\title{
First Experimental Mach-Zehnder FOPA for Polarization- and Wavelength-Division-Multiplexed Signals
}

\author{
Florent Bessin (1), Vladimir Gordienko(1), Filipe M. Ferreira(2), Nick Doran ${ }^{(1)}$ \\ (1) Aston Institute of Photonic Technologies, Aston University, Birmingham, B4 7ET, England, \\ (2) Optical Networks Group, University College London, London, WC1E 6BT, UK \\ bessinf@aston.ac.uk
}

\begin{abstract}
We demonstrate and characterize a polarization insensitive fiber optical parametric amplifier based on a Mach-Zehnder configuration using a commercial 100G PDM-QPSK transponder. A net gain $>10 \mathrm{~dB}$ is obtained for 17 channels in C-band, $100 \mathrm{GHz}$ spaced.
\end{abstract}

\section{Introduction}

Fiber optical parametric amplifiers (FOPA) have been widely studied for a few decades due to their abilities to provide wide bandwidth gain [1], ultra-low noise figure ${ }^{[2]}$ and arbitrary wavelength range operation ${ }^{[3]}$. However, one of the major issues of FOPA is their polarization dependent gain. To overcome this issue, different technics have been investigated such as half pass loop FOPA that as demonstrate great results ${ }^{[4]}$. In this configuration both polarization components of the input signal propagate in opposite direction in the FOPA loop. Thus, each polarization of the input signal can be amplified independently. Nevertheless, these signal components pass first through a loss section accumulating small nonlinear crosstalk and then through a gain section, or vice-versa - with stronger nonlinear crosstalk limiting the FOPA performance ${ }^{[5]-[6]}$.

In this paper, we demonstrate for the first time to our knowledge a promising polarization insensitive (PI-)FOPA based on a MachZehnder (MZ) architecture that gets rid of these passive sections, inherent to half pass loop FOPA and responsible for losses and crosstalk.
Indeed, in MZ configuration an input signal is first split into two single-polarization components, each one propagating in one of the two arms of the $M Z$ (each containing an independent gain section), and finally recombined. We show in particular that we can amplify DWDM signals in C-band with a gain $>10 \mathrm{~dB}$, using signal amplification of 100G PDMQPSK signals supplied by a Ciena transponder WaveLogic 3.

\section{Experimantal setup}

Figure 1 shows the in line transmission arrangement test bed that consists in a transmitter (a commercial transponder plus ASE-shaped channels), a variable optical attenuator to set input power, our PI-FOPA and a receiver.

The PI-FOPA is based on Mach-Zehnder interferometer architecture. The signal is firstly guided through a polarisation beam splitter (PBS1) that separates input signal into two single polarization components (Signal $x / y$ ). Each component then undergoes a gain section of highly nonlinear fiber (HNLFXYY, respectively)

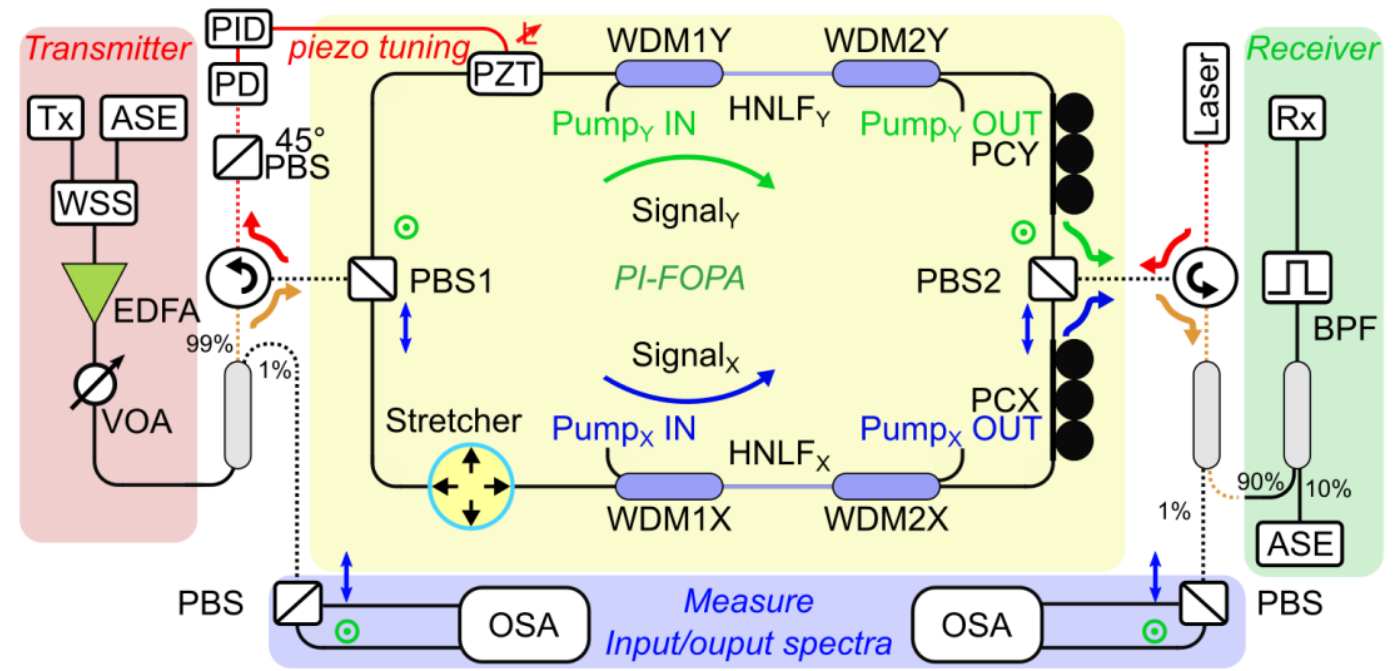

Fig. 1: Experimental setup of a polarization insensitive Mach-Zehnder FOPA. OSA: optical spectrum analyzer; PD, photodetector; PBS, polarization beam splitter; PID, proportional-integrate-derivative; ASE, amplified spontaneous emission; PZT, piezo-electric acuator; HNLF, highly nonlinear fiber; EDFA, erbium doped fiber; VOA, variable optical attenuator. 
provided coupling with a $34.5 \mathrm{dBm}$ and 34.2 $\mathrm{dBm}$ co-propagating pump, for Signalx/Y respectively (PumpX/Y, respectively). Each HNLF length is $100 \mathrm{~m}$ long with zero-dispersion wavelength of $\sim 1551 \mathrm{~nm}$ and nonlinearity coefficient of $\sim 14 \mathrm{~W}^{-1} \cdot \mathrm{km}^{-1}$. Coupling of pumps and signals into HNLFs are performed by 200 $\mathrm{GHz}$-wide wavelength division multiplexing filters (WDM1XY) centered at $1553.33 \mathrm{~nm}$. In the same way, we use at the output of HNLFs WDM2X/y to remove pumps. Pumpx/y are obtained from a $100 \mathrm{kHz}$-linewidth tuneable laser source set to $\lambda_{p}=1553.38 \mathrm{~nm}$ and phase modulated to mitigate stimulated Brillouin scattering in the HNLF - high power is achieved using two erbium doped fiber amplifiers (EDFAxy) after a 50/50 splitter. Electronically addressable polarization controllers are used to align the pump and signal field polarizations to maximize the coupling efficiency. Finally, signal components are recombined by PBS2. We added in each arm a polarization controller (PCX and PCY) used to align Signalxy with PBS2 polarization axis for an efficient recombination.

An optical path difference between arms may cause a delay between Signal $X Y$ at the output of MZ-FOPA and leads to signal distortion. To match the optical paths, we cut-back fiber from the longer $M Z$ arm to a precision of a few centimeters. A mechanical stretcher embedded in arm-X allows to finely tune its length and match the optical paths between both arms. Due to the interferometric nature of this device, this system is extremely sensitive to external perturbations such as a change in pressure or temperature that induces variations of length between arms. To overcome this issue we added circulators at each sides of MZ-FOPA similar to Ref. ${ }^{[7]}$ allowing us to use independently both direction of propagation. While data signal propagates from left to right, a reference signal at $\lambda_{\text {ref }}=1567 \mathrm{~nm}$ propagates in the other direction. This reference signal, also affected by external perturbations, passes through a PBS with polarization axis at $45^{\circ}$ from those of PBS1. This arrangement ensures to observe the interference behavior of the reference signal with a high visibility via a photodetector. Then, we use this detected signal as an error signal for a feedback loop system (proportional integrate derivative) that drives a piezo-electric fiber stretcher embedded in arm- $Y$ and compensate for length fluctuations.

The optical transmitter of the test bed consisted of $17 \times 100 \mathrm{GHz}$ spaced channels in a wavelength range of $1528.0-1540.56 \mathrm{~nm}$. The channel at $1534.25 \mathrm{~nm}$ was a $100 \mathrm{G}$ PDM-QPSK signal sourced from a Ciena transponder WaveLogic 3. Other channels were emulated copies of the transponder sourced from amplified spontaneous emission (ASE) shaped by a wavelength selective switch (WSS). A VOA after transmitter was used to sweep power per channel between $-27 \mathrm{dBm}$ and $0 \mathrm{dBm}$.

The receiver coupled broadband ASE with signal via a 90/10 coupler allowing to sweep OSNR, and selected the 100G channel using a band-pass filter (BPF). This filtered channel was then coherently detected by a Ciena transponder WaveLogic 3.

Note that in order to measure input and ouput spectra of both signal polarization components, cirulators and third ports of PBS1 and PBS2 are made of polarization maintening (PM) fibers (dotted lines in Fig. 1). Thus, adding 99/1 PM tap couplers before and after FOPA, we can obtain these spectra through $1 \%$ ports thanks to PBS.

\section{Experimental Results}

Figure 2 (a) and (b) show the FOPA input and ouput DWDM spectra for $\mathrm{X}$ and $\mathrm{Y}$ signal polarization components respectively, when power per channel was set to $-23 \mathrm{dBm}$ at FOPA input and we observed a power per channel >$12 \mathrm{dBm}$ at FOPA output. We also noted a residual pump power (not shown here) of about $10 \mathrm{dBm}$ which was not detrimental in our experiment since it was filtered before receiver by the BPF tuned to the 100G channel wavelength.

Figure 3 depicts the net gain for each channel

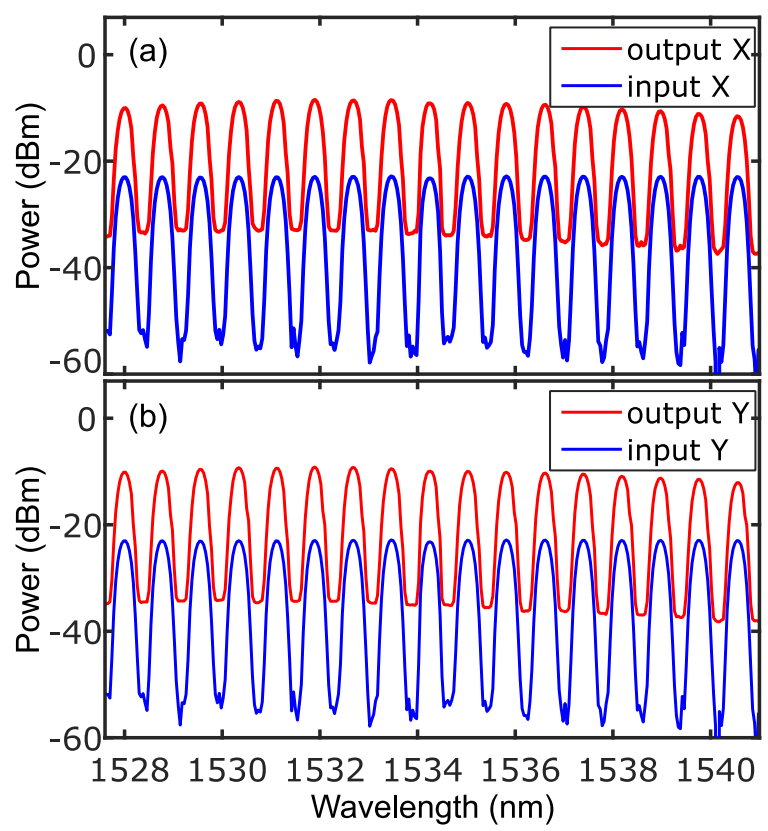

Fig. 2: The blue and red curves depict the signal input and output power for $X$ and $Y$ polarization components in (a) and (b), respectively. 
in blue and red for $\mathrm{X}$ and $\mathrm{Y}$ components, respectively. The net gain for polarization components for the $100 \mathrm{G}$ channel at $1534.25 \mathrm{~nm}$ is about $12.6 \mathrm{~dB}$ and in a range of 10.5 and $13.3 \mathrm{~dB}$ for other channels. The black dots in Fig. 3 correspond to the polarization dependent gain (PDG) of each channel. It is about $0.01 \mathrm{~dB}$ for $100 \mathrm{G}$ channel and in a range of $\pm 0.5 \mathrm{~dB}$ for other channels.

In Fig. 4, we display the bit error rate as

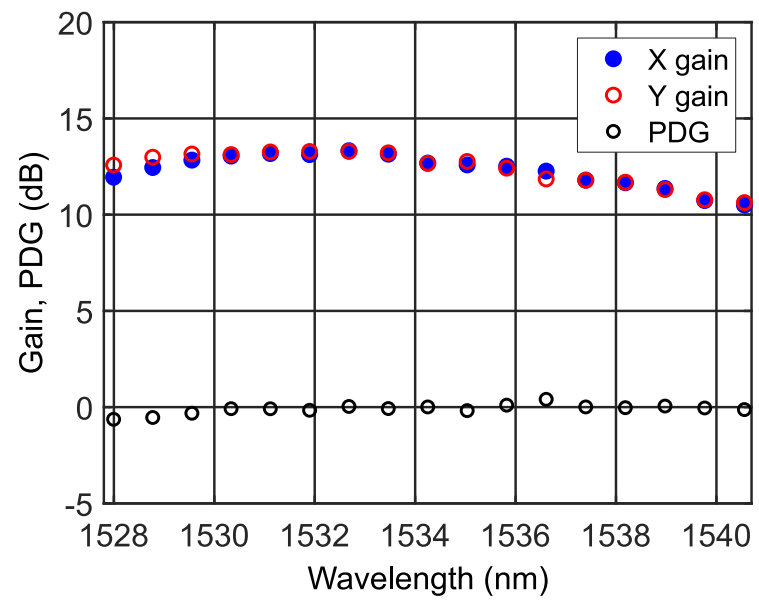

Fig. 3: FOPA net gain for each channel. The blue and red circles depict net gain of $\mathrm{X}$ and $\mathrm{Y}$ signal polarization components, respectively. The black circles depict the polarization dependence gain for each channel.

function of the optical power at the receiver for the 100G channel. These results show promising results with a BER of $10^{-8}$ for a received power of $-3 \mathrm{dBm}$. We also note for a fixed received $B E R$ of $10^{-3}$, that penalty on the receiver sensitivity is about $1.9 \mathrm{~dB}$.

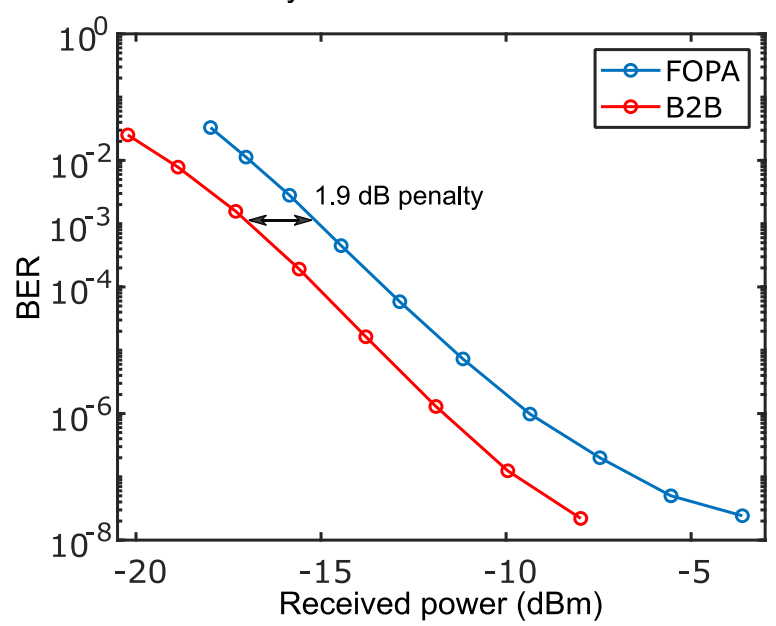

Fig. 4: Bit error rate versus received power. The red curve stands for back to back measurements and the blue curve for FOPA measurements.

\section{Conclusions}

We provide the first experimental demonstration of a PI-FOPA based on a Mach-Zehnder configuration to amplify $100 \mathrm{G}$ PDM-QPSK signal. We obtained a net gain $>10 \mathrm{~dB}$ for both signal polarization components for emulated DWDM from 1528.0 to $1540.56 \mathrm{~nm}$. These results provide a proof of concept of such amplifier architecture and open the way to future improvement about crosstalk and losses in FOPA.

\section{Acknowledgements}

We wish to thank Dr Shigehiro Takasaka and Dr Ryuichi Sugizaki of Furukawa Electric for highly nonlinear fibers, and $\mathrm{Dr}$ Charles Laperle of Ciena for the transponder. This work was supported in part by UK EPSRC for project UPON EP/M005283/1, FPA-

ROCS EP/R024057/1 (Reasearch data: https://doi.org/10.17036/researchdata.aston.ac.u k.00000500), H2020 Marie Skłodowska-Curie Actions (MSCA)(713694) and the UKRI Future Leaders Fellowship under Grant MR/T041218/1.

\section{References}

[1] V. Gordienko, M. F. C. Stephens, A. E. El-Taher, and N. J. Doran, Ultra-Flat Wideband Single-Pump RamanEnhanced Parametric Amplification, Opt. Express, OE 25, 4810 (2017).

[2] Z. Tong, C. Lundström, P. A. Andrekson, C. J. McKinstrie, M. Karlsson, D. J. Blessing, E. Tipsuwannakul, B. J. Puttnam, H. Toda, and L. GrünerNielsen, Towards Ultrasensitive Optical Links Enabled by Low-Noise Phase-Sensitive Amplifiers, Nature Photonics 5, 7 (2011).

[3] V. Gordienko, M. F. C. Stephens, and N. J. Doran, Raman-Generated Pump and Its Use for Parametric Amplification and Phase Conjugation, in 2018 European Conference on Optical Communication (ECOC) (2018), pp. 1-3.

[4] M. F. C. Stephens, V. Gordienko, and N. J. Doran, 20 $D B$ Net-Gain Polarization-Insensitive Fiber Optical Parametric Amplifier with \&gt;2 THz Bandwidth, Opt. Express, OE 25, 10597 (2017).

[5] V. Gordienko, F. Ferreira, C. Laperle, M. O'Sullivan, C. B. Gaur, K. Roberts, and N. Doran, Noise Figure Evaluation of Polarization-Insensitive Single-Pump Fiber Optical Parametric Amplifiers, in Optical Fiber Communication Conference (OFC) 2020 (2020), Paper W4B.4 (Optical Society of America, 2020), p. W4B.4.

[6] M. F. C. Stephens, V. Gordienko, and N. J. Doran, Reduced Crosstalk, Polarization Insensitive Fiber Optical Parametric Amplifier (PI FOPA) for WDM Applications, in Optical Fiber Communication Conference (2018), Paper W3D.4 (Optical Society of America, 2018), p. W3D.4.

[7] F. Bessin, F. Copie, M. Conforti, A. Kudlinski, A. Mussot, and S. Trillo, Real-Time Characterization of Period-Doubling Dynamics in Uniform and Dispersion Oscillating Fiber Ring Cavities, Phys. Rev. X 9, 041030 (2019). 